\title{
Indicators of Mass in Spherical Stellar Atmospheres
}

\author{
John B. Lester \\ Department of Chemical and Physical Sciences, University of Toronto Mississauga, \\ Mississauga, Ont, L5L 1 C6 Canada \\ and \\ Department of Astronomy \& Astrophysics, University of Toronto \\ lester@astro.utoronto.ca \\ Rayomond Dinshaw \\ Department of Chemistry, University of Toronto, Toronto, Ont, M5S 3H6 Canada \\ rdinshaw@chem.utoronto.ca \\ and \\ Hilding R. Neilson \\ Department of Physics $\&$ Astronomy, East Tennessee State University, Box 70652, \\ Johnson City, TN, 37614, USA \\ neilsonh@etsu.edu
}

\begin{abstract}
Mass is the most important stellar parameter, but it is not directly observable for a single star. Spherical model stellar atmospheres are explicitly characterized by their luminosity $\left(L_{\star}\right)$, mass $\left(M_{\star}\right)$ and radius $\left(R_{\star}\right)$, and observations can now determine directly $L_{\star}$ and $R_{\star}$. We computed spherical model atmospheres for red giants and for red supergiants holding $L_{\star}$ and $R_{\star}$ constant at characteristic values for each type of star but varying $M_{\star}$, and we searched the predicted flux spectra and surface-brightness distributions for features that changed with mass. For both stellar classes we found similar signatures of the star's mass in both the surface-brightness distribution and the flux spectrum. The spectral features have been use previously to determine $\log _{10}(g)$, and now that the luminosity and radius of a non-binary red giant or red supergiant can be observed, spherical
\end{abstract}


model stellar atmospheres can be used to determine the star's mass from currently achievable spectroscopy. The surface-brightness variations with mass are slightly smaller than can be resolved by current stellar imaging, but they offer the advantage of being less sensitive to the detailed chemical composition of the atmosphere.

Subject headings: stars:atmospheres - stars:fundamental parameters - stars:latetype - stars:supergiants

\section{Introduction}

Plane-parallel model stellar atmospheres are characterized by their effective temperature, defined as

$$
T_{\text {eff }} \equiv\left(\frac{L_{\star}}{\sigma 4 \pi R_{\star}^{2}}\right)^{1 / 4},
$$

and surface gravity,

$$
g \equiv G \frac{M_{\star}}{R_{\star}^{2}} .
$$

As is apparent, these two parameters are degenerate expressions of the three more fundamental stellar parameters $L_{\star}, M_{\star}$ and $R_{\star}$. Along the main sequence the variation of $R_{\star}$ is small, making $T_{\text {eff }}$ and $\log _{10}(g)$ nearly unique identifiers of the star. However, in the cool, luminous quadrant of the Hertzsprung-Russell diagram, stars with a large range of $R_{\star}$ and $M_{\star}$ converge to similar values of $T_{\text {eff }}$ and $\log _{10}(g)$, which makes it difficult to establish the fundamental parameters of non-binary red giants and red supergiants.

Fortunately, optical/infrared interferometry is helping to break this degeneracy by measuring directly the angular diameters of stars. By combining these angular diameters with stellar parallaxes that are being measured with increasing precision to ever greater distances, it is now possible to determine $R_{\star}$ observationally. In addition, combining the stellar flux measured at Earth with the same stellar angular diameter and parallax yields the $L_{\star}$, assuming the star radiates isotropically. With measured values of $L_{\star}$ and $R_{\star}$, the observational attention turns to $M_{\star}$, the key parameter that determines the other two.

It is now routine (Hauschildt et al. 1999; Gustafsson et al. 2008; Lester \& Neilson 2008) to compute spherically symmetric models of stellar atmospheres that are characterized explicitly by $L_{\star}, M_{\star}$ and $R_{\star}$, in addition to the chemical composition. Assuming that red giants and supergiants are spherically symmetric, the ability to measure $L_{\star}$ and $R_{\star}$ raises the question whether spherical stellar atmospheres predict any indicators of stellar mass that could be determined observationally. 


\section{Red Giants}

To search for possible indicators of stellar mass, the SATLAS program (Lester \& Neilson 2008) was used to compute a spherical model atmosphere with properties representative of a typical red giant: $L_{\star}=500 L_{\odot}, M_{\star}=0.8 M_{\odot}$ and $R_{\star}=50 R_{\odot}$. The model was calculated with the opacity distribution function (ODF) version of SATLAS using the ODFs recomputed by Castelli (wwwuser.oat.ts.astro.it/castelli/) in 2012 February to incorporate Kurucz's corrections to his $\mathrm{H}_{2} \mathrm{O}$ line list. As Kurucz explains (kurucz.harvard.edu/molecules/H2O/h2ofastfix.readme), he uses the $g f$ values from Partridge \& Schwenke (1997) after renormalizing them to agree with the $g f$ values from the HITRAN (HIgh-resolution TRANsmission) molecular absorption database (www.cfa.harvard.edu/hitran/), but ignoring nuclear spin factors. Kurucz's original $\mathrm{H}_{2} \mathrm{O}$ line list did not take into account that the $\mathrm{H}_{2}^{16} \mathrm{O}, \mathrm{H}_{2}^{17} \mathrm{O}, \mathrm{H}_{2}^{18} \mathrm{O}$ and $\mathrm{HD}^{16} \mathrm{O}$ isotopologs each has its own partition function. To correct for this, while also using only the $\mathrm{H}_{2}^{16} \mathrm{O}$ partition function in Atras, Kurucz assumes that, at stellar temperatures, the partition function of each isotopolog differs from the partition function of $\mathrm{H}_{2}^{16} \mathrm{O}$ by a constant multiplicative factor, which he determines by comparing the $g f$ values of a few strong lines in the HITRAN database with the Partridge \& Schwenke (1997) values. The ODF assumes a depth-independent microturbulent velocity of $2 \mathrm{~km} / \mathrm{s}$ and the solar abundances tabulated by Grevesse \& Sauval (1998). The mean difference between the Grevesse \& Sauval (1998) abundances and the more recent tabulation of Asplund et al. (2009) for the elements with number densities $\geq 10^{-10} \times N_{\mathrm{H}}$ is only 0.02 in $\log _{10}$ of the abundances. The ODF also uses the large wavelength spacing with an average spectral resolving power of $R \equiv \lambda / \Delta \lambda \approx 50$, corresponding to $\Delta \lambda \approx 10 \mathrm{~nm}$ at $500 \mathrm{~nm}$. A test using an ODF with the smaller wavelength space, which gives $\approx 5 \times$ higher spectral resolving power, produced a model structure (temperature as a function of gas pressure) that was almost indistinguishable from the structure of the model with the lower resolution ODF. Therefore, the lower resolution ODF was used to calculate all the models. The model was iterated until the luminosity was constant to $\leq 1 \%$ for $\log _{10}\left(\tau_{\text {Ross }}\right) \geq 10^{-7}$ and the derivative of the luminosity was constant to $\leq 1 \%$ for $\log _{10}\left(\tau_{\text {Ross }}\right) \geq 10^{-3}$, where $\tau_{\text {Ross }}$ is the Rosseland optical depth.

To test the dependence on mass, two additional red giant models were computed holding the values of $L_{\star}$ and $R_{\star}$ constant, but with $M_{\star}=1.6 M_{\odot}$ and $M_{\star}=2.4 M_{\odot}$. These parameters correspond to $T_{\text {eff }}=3865 \mathrm{~K}$ for all three stars, but $\log _{10}(g)=0.94$ for the $0.8 M_{\odot}$ star, $\log _{10}(g)=1.24$ for the $1.6 M_{\odot}$ star and $\log _{10}(g)=1.42$ for the $2.4 M_{\odot}$ star. The effect of mass on the stellar atmosphere can be represented by a relative extension parameter, $\epsilon$, defined as the radial distance from the deepest visible layer of the atmosphere out to an arbitrary upper radius, normalized by the stellar radius,

$$
\epsilon \equiv \frac{R(\text { upper })-R(\text { deep })}{R_{\star}}
$$


A convenient value for $R$ (deep) is where $\tau_{\text {Ross }}=1$, and the radiation's probability of escape is $\exp (-1)=0.36$. The definition of the upper radius is arbitrary. Defining $R$ (upper) where $\tau_{\text {Ross }}=-3$, corresponding to about three pressure scale heights, leads to $\epsilon(-3)$. Using this, the $0.8 M_{\odot}$ star has $\epsilon(-3) \approx 0.025$, the $1.6 M_{\odot}$ star has $\epsilon(-3) \approx 0.012$ and the $2.4 M_{\odot}$ star has $\epsilon(-3) \approx 0.008$. Defining the upper $R$ is where $\tau_{\text {Ross }}=-6$, leads to the definition of $\epsilon(-6)$. Using this, the $0.8 M_{\odot}$ star has $\epsilon(-6) \approx 0.06$, the $1.6 M_{\odot}$ star has $\epsilon(-6) \approx 0.028$ and the $2.4 M_{\odot}$ star has $\epsilon(-6) \approx 0.019$. Clearly the extension parameter is greater with this choice of $R$ (upper), but by either definition these test models have modest extension.

\subsection{Surface Brightness Distribution}

The three red giant model atmospheres were used to calculate the surface intensity at 100 values of the disk's fractional radius, $r / R_{\star}$, ranging from 0 to 1 in steps of 0.01 , for each wavelength bin of the ODF that had a non-negligible amount of radiation. A comparison was made of the surface brightness distributions of the same model parameters computed with both the lower and higher spectral resolution ODFs described in the previous section. At wavelengths $\gtrsim 1000 \mathrm{~nm}$, the surface brightness distributions were almost identical for the two spectral resolutions because there are so few spectral lines. At wavelengths $<1000 \mathrm{~nm}$, the surface brightness distributions do differ very slightly because there are more and stronger spectral lines. The ODF with the finer wavelength spacing resolves some spectral detail that is averaged out in the lower resolution ODF. As a result, at some wavelengths the surface brightness distribution is shifted slightly toward or away from $R_{\star}$, depending on whether the higher resolution ODF happens to include a strong line or not. The lower spectral resolution surface brightness distribution are used here because they seem to be a better match to the observations, but the synthetic spectra described in $\S 2.2$ can compute the surface brightness distribution for any spectral resolving power, which can match any particular observation exactly. Figure 1 shows the limb darkening curves for the three red giant models at the ODF wavelengths close to the effective wavelengths of the $V$ and $R$ magnitudes listed by Bessell (2005) and to the effective wavelengths of the $I, J, H$ and $K$ magnitudes given by Tokunaga (2000). It is apparent that increasing the stellar mass makes the relative intensity greater at every location on the stellar disk for every wavelength. This trend results from the gas density and the thermal radiation of the atmosphere increasing with the stellar mass. It is also apparent that the curves flatten as the wavelength increases toward the peak of the Planck distribution for the star's temperature.

The differences between the curves in Figure 1 uses a knowledge of the stellar limb's absolute location, $R_{\star}$, which is present in the models. However, as the stellar mass is 


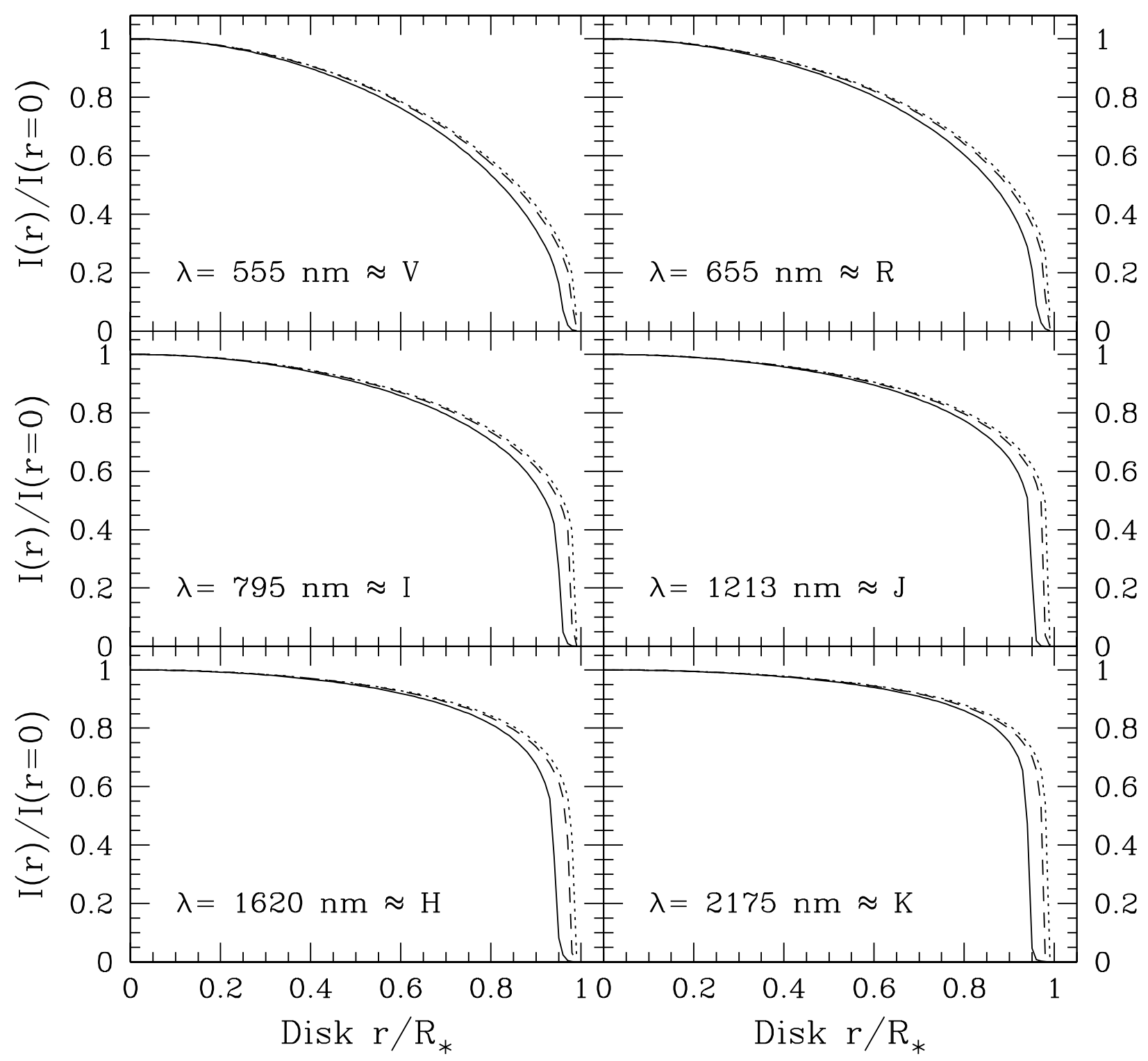

Fig. 1. - Surface brightness distributions for three red giant spherical model atmospheres at several wavelengths. The atmospheres all have $L_{\star}=500 L_{\odot}$ and $R_{\star}=50 R_{\odot}$. The solid lines are for $M_{\star}=0.8 M_{\odot}$, the dashed lines are for $M_{\star}=1.6 M_{\odot}$ and the dotted lines are for $M_{\star}=2.4 M_{\odot}$. The wavelengths are approximately the effective wavelengths of the $V$, $R, I, J, K$ and $H$ filter systems. 
reduced, the upper atmosphere becomes more tenuous, and the radiation from these outer radii decreases to the point where some of the curves in Figure 1 appear to go to zero before reaching $R_{\star}$. In fact, the relative intensity is not zero, but it is less than 0.01 , making it too small to be resolved in the plot. This reduced relative intensity at the stellar limb is a direct consequence of the spherical geometry of the models, and it also affects the observations.

To recast the differences in Figure 1 into a form that is suitable for observational application, we define the location of the stellar limb to be where the surface brightness has fallen to a specified fraction of the central brightness,

$$
R_{\mathrm{limb}} \equiv r \text { where } \frac{I(r)}{I(r=0)}=f
$$

Using this definition of $R_{\text {limb }}$, we renormalize the surface brightness distribution. Figure 2 shows the result of setting $f=0.01,0.02,0.05$ and 0.1 for one wavelength of one spherical model. The wavelength $\lambda=705 \mathrm{~nm}$ is approximately in the middle of the range shown in Figure 1. From an examination of Figure 2 we elect to define $R_{\text {limb }}$ using $f=0.01$. This choice gives a value of $R_{\text {limb }}$ that is close to $R_{\star}$ while also being within reach of observations, but clearly another value of $f$ could be chosen. Figure 1 shows that the surface brightness falls off more steeply at the limb as the model's mass increases, which reduces the difference between $R_{\text {limb }}$ and $R_{\star}$. With an angular resolution $\approx 1 \%$ of the stellar disk and a photometric precision of $\approx 1 \%$, observations of the surface-brightness distribution from optical interferometry or a planetary transit could determine this location of the stellar limb.

Using this observationally motivated definition of the stellar limb, we renormalized the intensity curves at each ODF wavelength and searched for the fractional radii where stellar mass produced the largest intensity difference. As an example, Figure 3 shows the differences between the $r / R_{\text {limb }}$ values for the red giant models with $0.8 M_{\odot}$ and $1.6 M_{\odot}$ and between 1.6 $M_{\odot}$ and $2.4 M_{\odot}$ models for the various limb renormalizations considered in Figure 2 , The wavelength $\lambda=705 \mathrm{~nm}$ is typical of a wide range of wavelengths. The difference between the $1.6 M_{\odot}$ and $2.4 M_{\odot}$ models is nearly the same for all the limb renormalizations, with the difference being greatest near $I(r) / I(r=0) \approx 0.5$. The difference between the $0.8 M_{\odot}$ and $1.6 M_{\odot}$ models is also greatest near $I(r) / I(r=0) \approx 0.5$ for the renormalization at $f=0.01$, but as the renormalization point is located farther in from $R_{\star}$, the location of the greatest difference between the two models also moves inward. We elect to use the difference at $I(r) / I(r=0)=0.5$ because it applies to the limb normalization with $f=0.01$ and because the half-intensity point is easy to determine observationally.

We determined the renormalized fractional stellar radius, $r / R_{\text {limb }}$, at the half-intensity point for each ODF wavelength from $500 \mathrm{~nm}$ to $2000 \mathrm{~nm}$, which is shown in Figure 4. The models show similar trends with wavelength. For $\lesssim 900 \mathrm{~nm}$ the half-intensity point moves 


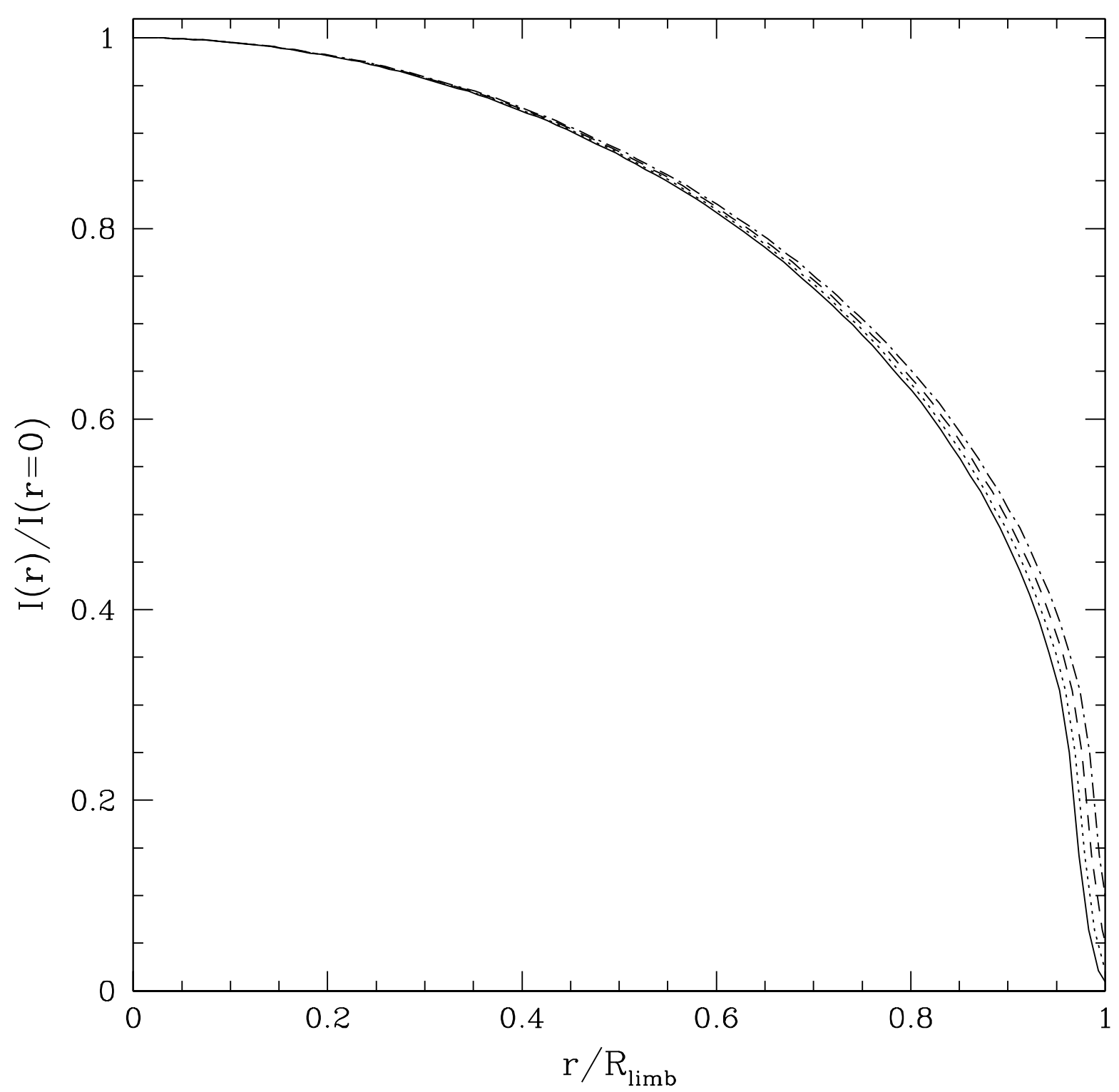

Fig. 2.- Renormalization of the limb-darkening profile at $\lambda=705 \mathrm{~nm}$ for the spherical model atmosphere with $L_{\star}=500 L_{\odot}, R_{\star}=50 R_{\odot}$ and $M_{\star}=0.8 M_{\odot}$. The different curves represent different choices for the value of " $f$ " in Equation 4 to define $R_{\text {limb }}$. The solid line is for $f=0.01$, the dotted line is for $f=0.02$, the dashed line is for $f=0.05$ and the dot-dash line is for $f=0.1$. 


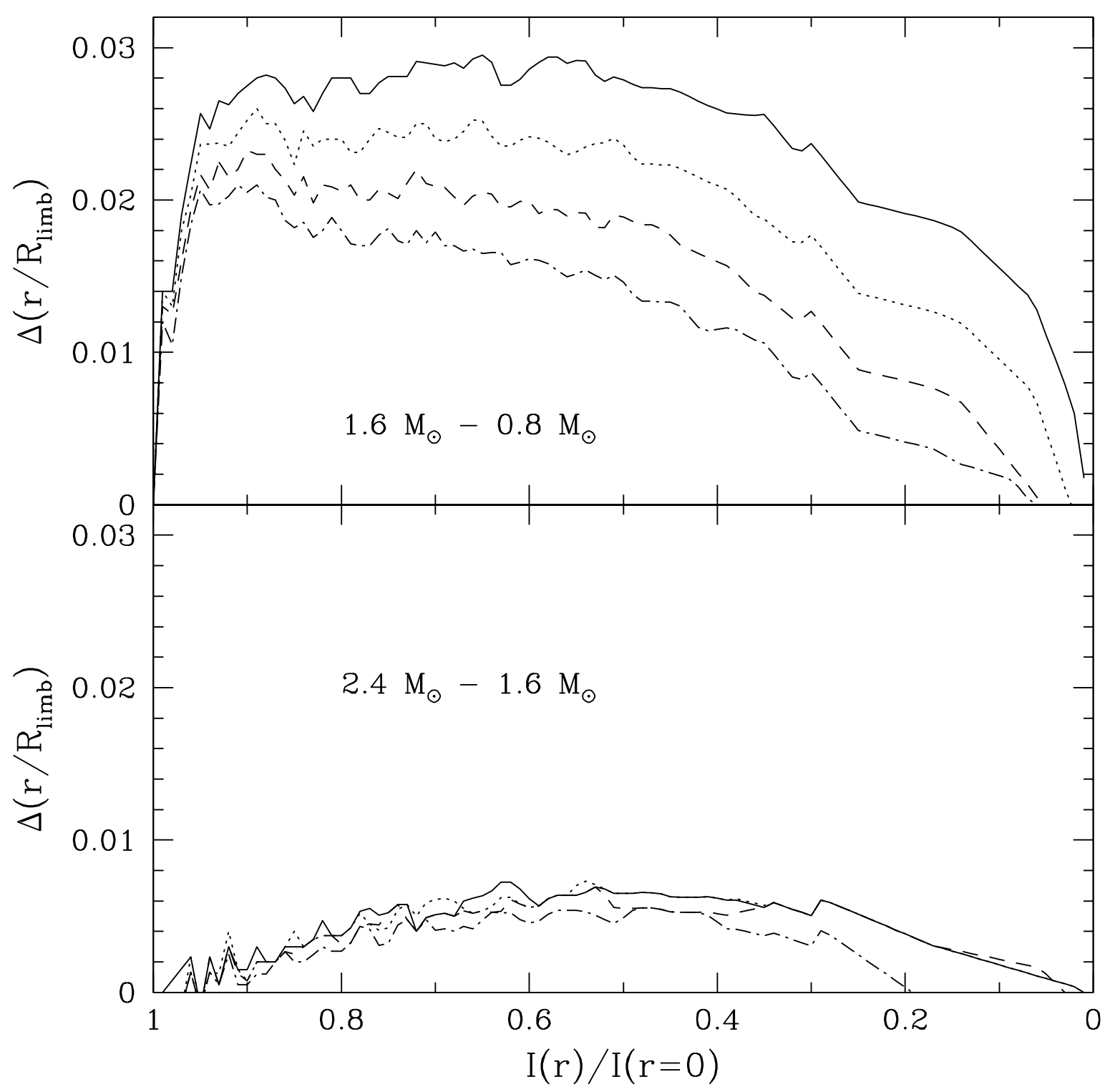

Fig. 3.- Difference between $r / R_{\text {limb }}$ at $\lambda=705 \mathrm{~nm}$ plotted as a function of the renormalized surface-brightness distribution. The solid line is for the limb defined using Equation 4 with $f=0.01$, the dotted line is for $f=0.02$, the dashed line is for $f=0.05$ and the dot-dash line is for $f=0.1$. The top plot shows the difference between the $0.8 M_{\odot}$ and $1.6 M_{\odot}$ models, and the bottom plot shows the difference between the $1.6 M_{\odot}$ and $2.4 M_{\odot}$ models. 


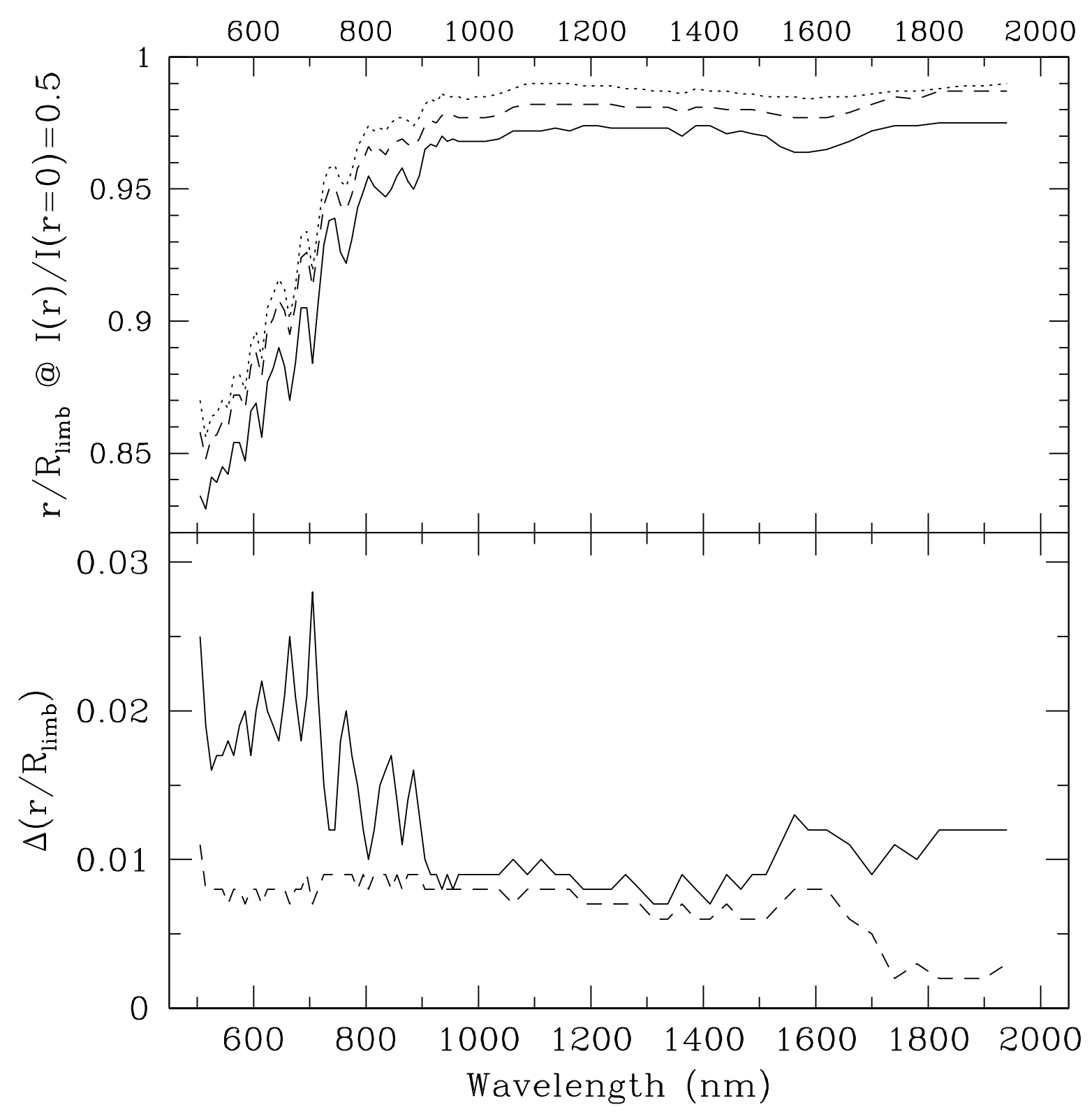

Fig. 4.- Top plot displays for the three red giant spherical models the wavelength dependence of the renormalized fractional radius $\left(r / R_{\text {limb }}\right)$ where the surface brightness is half the central brightness. The spherical models all have $L_{\star}=500 L_{\odot}$ and $R_{\star}=50 R_{\odot}$. The solid line is for $M_{\star}=0.8 M_{\odot}$, the dashed line is for $M_{\star}=1.6 M_{\odot}$ and the dotted line is for $M_{\star}=2.4 M_{\odot}$. Bottom plot is the differences between the models in the top panel, with the solid line being the difference between the $0.8 M_{\odot}$ and $1.6 M_{\odot}$ models and the dashed line is the difference between the $1.6 M_{\odot}$ and $2.4 M_{\odot}$ models. 
toward the limb as the wavelength increases, but for $\lambda>900 \mathrm{~nm}$ the fractional radius of the half-intensity point is nearly constant. Within these general trends the three masses are offset from each other, with the location of the half-intensity point shifting toward the limb as the mass increases. It is also clear from the bottom panel of Figure 4 that the location of the half-intensity point varies significantly with wavelength for the lowest mass model considered here. For example, the separation is relatively larger at $705 \mathrm{~nm}$, the wavelength used in Figure 2, than at adjacent wavelengths. The separation also increases near $1600 \mathrm{~nm}$, where the continuous opacity due to $\mathrm{H}^{-}$is minimum.

Examining the stellar spectrum in the regions of larger separation, we find that they have relatively less line opacity. Because of this, the intensity at these wavelengths comes from the thermal emission deeper in the atmosphere. As the mass of the star increases, the greater gas density produces a larger amount of thermal emission. As a result, the surfacebrightness distribution of the more massive star is flatter, and its half-intensity point shifts toward the limb. The conclusion is that it is important to measure the surface-brightness distribution with sufficient spectral resolution, $R \approx 50-100$, to isolate the most sensitive spectral windows. Of the existing interferometers capable of working in the optical region where the separation between the curves is greatest, the CHARA array's VEGA instrument (Mourard et al. 2009), which observes the band from 0.45 to $0.85 \mu \mathrm{m}$, has three choices of spectral resolution, $R=1700,6000$ and 30000, all of which are substantially greater than the required spectral resolving power. The Navy Precision Optical Interferometer (Armstrong et al. 1998) disperses the spectral band from 450 to $850 \mathrm{~nm}$ onto 32 channels, providing $R \sim 50$, just at the lower edge of the required spectral resolution. In the infrared, the AMBER instrument on the VLTI (Petrov \& AMBER Consortium 2003) could easily

resolve the $\mathrm{H}^{-}$feature. The bigger challenge for all the interferometers is achieving the angular resolution needed to locate the stellar disk's half-intensity point with an uncertainty of just a few percent. Although this appears to be beyond the current capability, the rapid progress being made in this active field makes the needed improvement seem likely.

\subsection{Spectrum}

Because the model's mass affects its structure, there can be associated variations of the model's spectral flux. To test this, we computed synthetic flux spectra covering the wavelength range from 400 to $1000 \mathrm{~nm}$ using the red giant spherical model atmospheres with $M_{\star}=0.8 M_{\odot}$ and $2.4 M_{\odot}$. These spectra, which were computed using spherical radiative transfer with the spherical model structure, have a spectral resolving power of $R=10^{5}$ and include the atomic, ionic and molecular lines from the lists on Kurucz's web 
site (http://kurucz.harvard.edu/linelists.html). Because we are comparing models to each other, not attempting to match observations, we have not included rotation or various kinds of broadening beyond the depth-independent microturbulent velocity of $2 \mathrm{~km} / \mathrm{s}$ that is used in the ODFs to compute the model atmospheres.

From a comparison of the synthetic spectra, we found that $\approx 95 \%$ of the lines either remained nearly constant or became weaker as the stellar mass increased. For the largest weakening, which occurred in the 400-500 nm wavelength band, the line core decreased by about 0.15 in residual intensity for the mass range of our models. The amount of line weakening diminished steadily with increasing wavelength, with our models in the 900$1000 \mathrm{~nm}$ interval having line core residual depths that were smaller by no more than about 0.07 .

At all wavelengths, the lines showing the greatest decrease with increasing mass were overwhelmingly ions of heavy elements, such as Nd II $536.1165 \mathrm{~nm}$ and La II $580.8313 \mathrm{~nm}$. However, there is one interesting exception to this dominance by heavy ions, which is [O I] ${ }^{3} \mathrm{P} 2 \mathrm{p}^{4} \rightarrow{ }^{1} \mathrm{D} 2 \mathrm{p}^{4}$ with lines at $630.0304 \mathrm{~nm}$ and $636.3776 \mathrm{~nm}$. Figure 5 shows the behavior of the $630.0304 \mathrm{~nm}$ line, which is consistent with the work of Bonnell \& Bell (1993b) who found that $[\mathrm{O} \mathrm{I}]$ is a useful indicator of surface gravity. Of course, $\log _{10}(g)$ combines $M_{\star}$ and $R_{\star}$, so it is a composite indicator, unlike our approach. For the $2.4 M_{\odot}$ star the equivalent width of [O I] $630.0304 \mathrm{~nm}$ is $23 \%$ less than for the $0.8 M_{\odot}$ star, and the decrease of the [O I] $636.3776 \mathrm{~nm}$ line is $31 \%$. As shown by Bonnell \& Bell (1993b), comparing measurements of these [O I] lines with lines of the $\mathrm{OH}$ molecule near $1.6 \mu \mathrm{m}$ yields the oxygen abundance and the surface gravity, or now the $M_{\star}$.

Of the $\approx 5 \%$ of the lines that became stronger with increasing mass, all of them were either $\mathrm{MgH}$ or $\mathrm{SiH}$, with $\mathrm{MgH}$ strengthening by about twice as much as $\mathrm{SiH}$. The largest increases in the residual line depth, about 0.08 for our models, were for lines of $\mathrm{MgH}$ in the $X^{2} \Sigma^{+} \rightarrow A^{2} \Pi$ band head around $500 \mathrm{~nm}$. An example of one line from this band is shown in Figure 6. where the line's equivalent width has become about $25 \%$ stronger going from the least to most massive model.

The lines of $\mathrm{MgH}$ in the $500 \mathrm{~nm}$ spectral region have a long history of serving as gravity indicators, beginning with Öhman (1934). More recently, Bell et al. (1985) and Bonnell \& Bell (1993a) have explored the use of these lines to determine the gravities of cool giants. By using the $\mathrm{MgH}$ lines in combination with lines of $\mathrm{Mg} \mathrm{I}$, it is possible to determine first the Mg abundance and then the surface gravity. Again, our focus on mass instead of the composite surface gravity allows for a sharper distinction.

As a test, we used the plane-parallel AtLAs_ODF code (Lester \& Neilson 2008) and the 


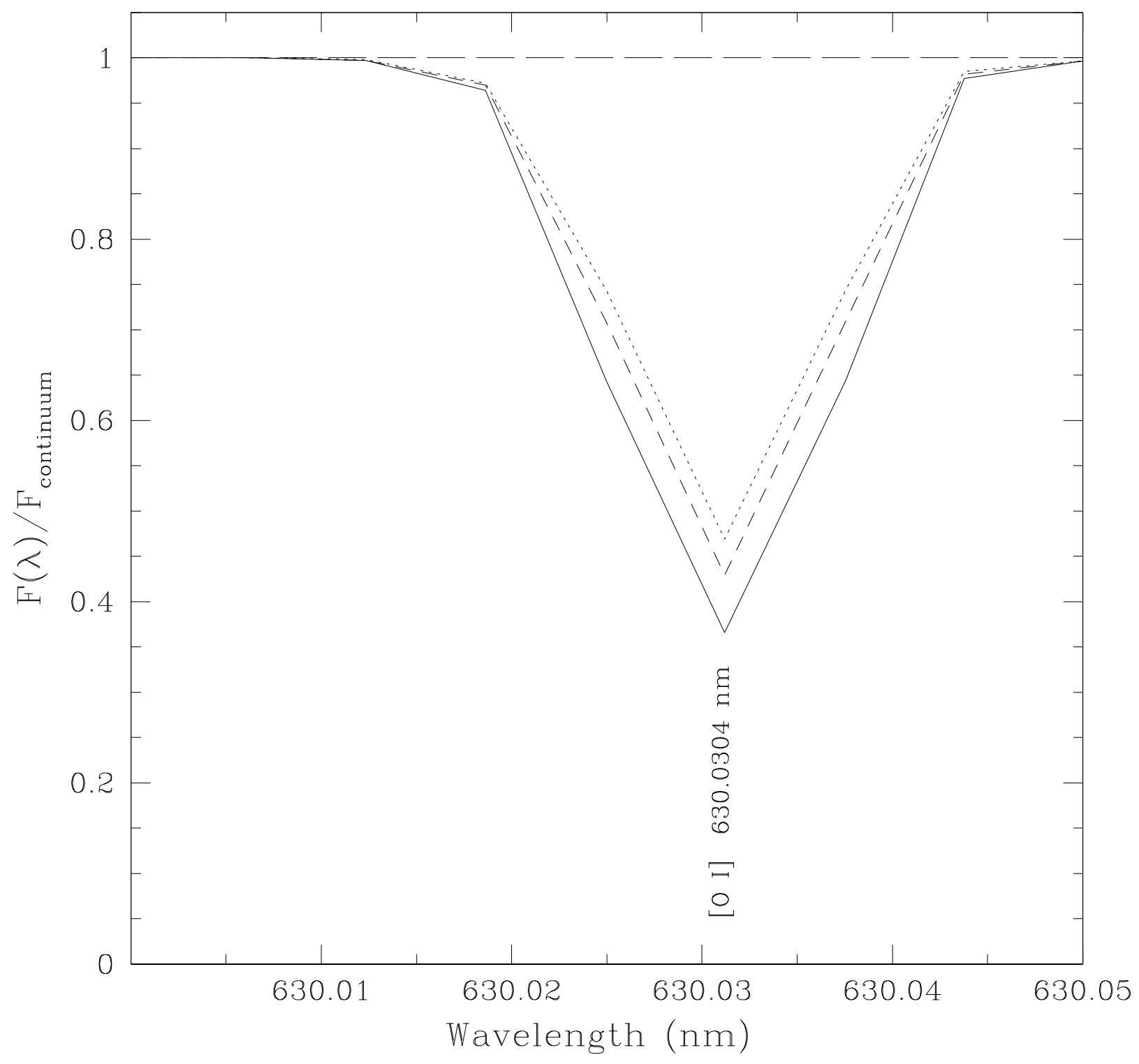

Fig. 5.- Rectified flux spectra of the line [O I] $\lambda=630.0304 \mathrm{~nm}$ for the red giant spherical models having $L_{\star}=500 L_{\odot}$ and $R_{\star}=50 R_{\odot}$. The solid line is for $M_{\star}=0.8 M_{\odot}$, the dashed line is for $M_{\star}=1.6 M_{\odot}$ and the dotted line is for $M_{\star}=2.4 M_{\odot}$. 


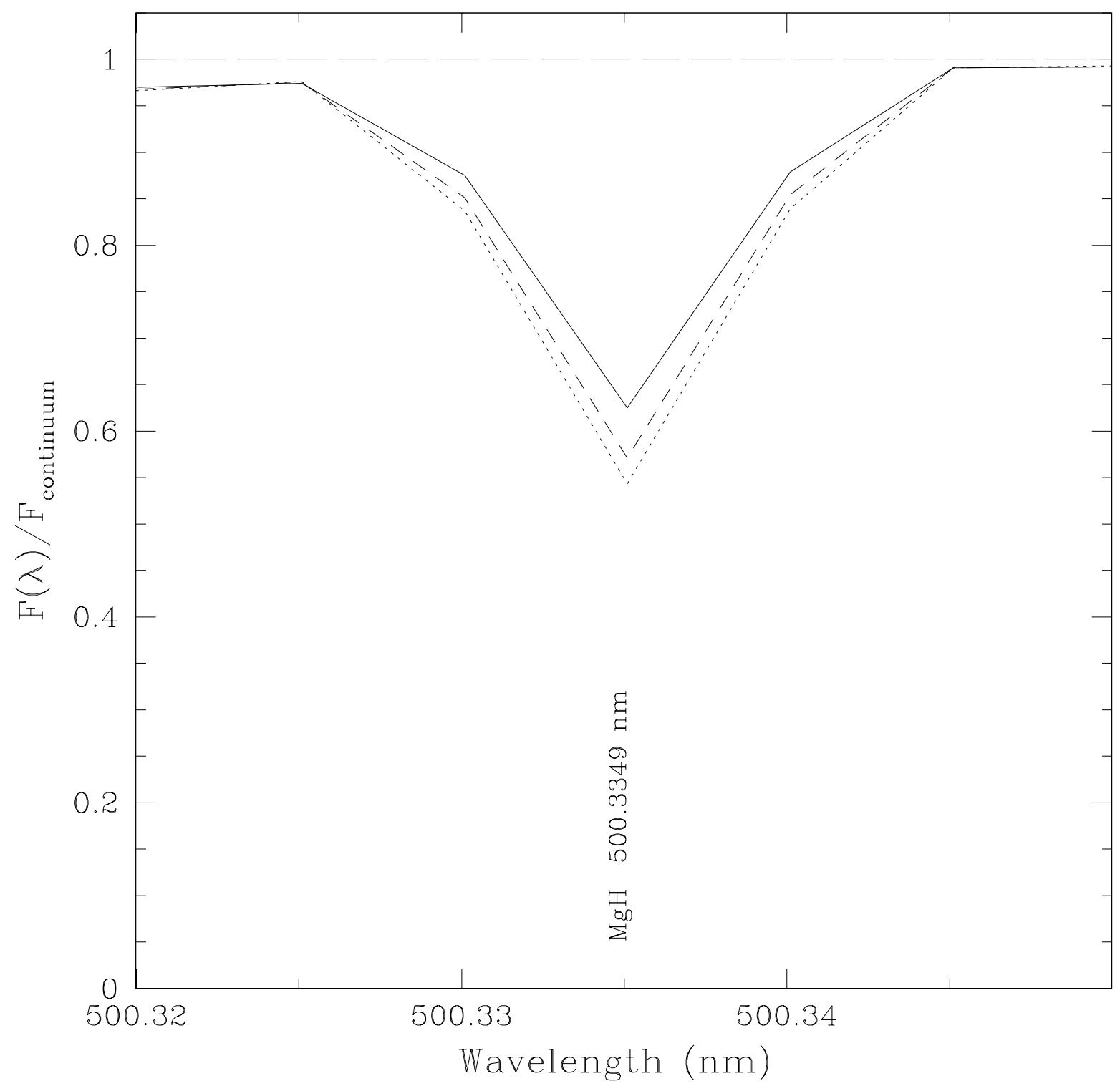

Fig. 6. - Rectified flux spectra of the $\mathrm{MgH}$ line at $\lambda=500.3349 \mathrm{~nm}$ for the red giant spherical models having $L_{\star}=500 L_{\odot}$ and $R_{\star}=50 R_{\odot}$. The solid line is for $M_{\star}=0.8 M_{\odot}$, the dashed line is for $M_{\star}=1.6 M_{\odot}$ and the dotted line is for $M_{\star}=2.4 M_{\odot}$. 
same ODF file to compute models with values of $T_{\text {eff }}$ and $\log _{10}(g)$ corresponding to the red giant spherical models. We used these plane-parallel models to compute synthetic spectra with a spectral resolving power of $10^{5}$ using the same line lists employed above. The resulting equivalent widths of the $[\mathrm{O} \mathrm{I}]$ and $\mathrm{MgH}$ lines, and their change with surface gravity, were nearly identical with values computed with the spherical model. This shows that these lines are sensitive to the mass, not the spherical geometry of the atmosphere.

The other molecular lines in the spectrum, except $\mathrm{SiH}$, show either almost no change or became weaker with increasing mass. To gain some perspective on this, we plot in the top panel of Figure 7 the number densities as a function of $\tau_{\text {Ross }}$ for several of the prominent diatomic molecules in the atmospheres of the red giant models with $M_{\star}=0.8 M_{\odot}$ and $2.4 M_{\odot}$. In the bottom panel of Figure 7 we show the variation of the temperature with $\tau_{\text {Ross }}$ for both models. The vertical dashed line in Figure 7 corresponds approximately to the atmospheric depth where the probability of radiation escaping to the observer becomes negligible. Note that only $\mathrm{MgH}$ and $\mathrm{SiH}$ have their peak number densities in the readily observed layers. This is a consequence of these two molecules having lower dissociation energies than any of the other common molecules, and $\mathrm{MgH}$, with a dissociation energy of

just $1.28517 \mathrm{eV}$ (Shayesteh et al. 2007), is more than a factor of two less than any of the other molecules.

\subsection{Composition}

The spectral results discussed in $\S 2.2$ clearly depend sensitively on the composition of the star. It is for this reason that it was necessary for Bonnell \& Bell (1993b) to use other spectral features to determine elemental composition before using either the [O I] or $\mathrm{MgH}$ lines to derive surface gravity. The surface-brightness distribution examined in $\S 2.1$ also depends on the metallicity of the star, which is shown in the left panel of Figure 8, As the metallicity is decreased from solar, the surface-brightness distribution becomes fainter at the stellar limb and brighter at $r / R_{\star} \lesssim 0.9$. Some of the relative intensities in the left panel of Figure 8 appear to go to zero before $r$ reaches $R_{\star}$. As explained earlier in the discussion of Figure 1, with decreasing stellar mass the upper atmosphere becomes increasingly tenuous, causing $\mathrm{I}(\mathrm{r}) / \mathrm{I}(\mathrm{r}=0)$ to drop below 0.01 as $r$ approaches $R_{\star}$. Even though the relative intensity is greater than zero, it is too small to be resolved in the plot.

The change at the limb is obvious for the models, for which $R_{\star}$ is known, but the location of $R_{\star}$ is not available observationally. Therefore, the same observational definition of the stellar limb introduced in $\S 2.1$ is employed here, with the result shown in the right panel of Figure 8. There is now a clear progression of the surface-brightness distribution 


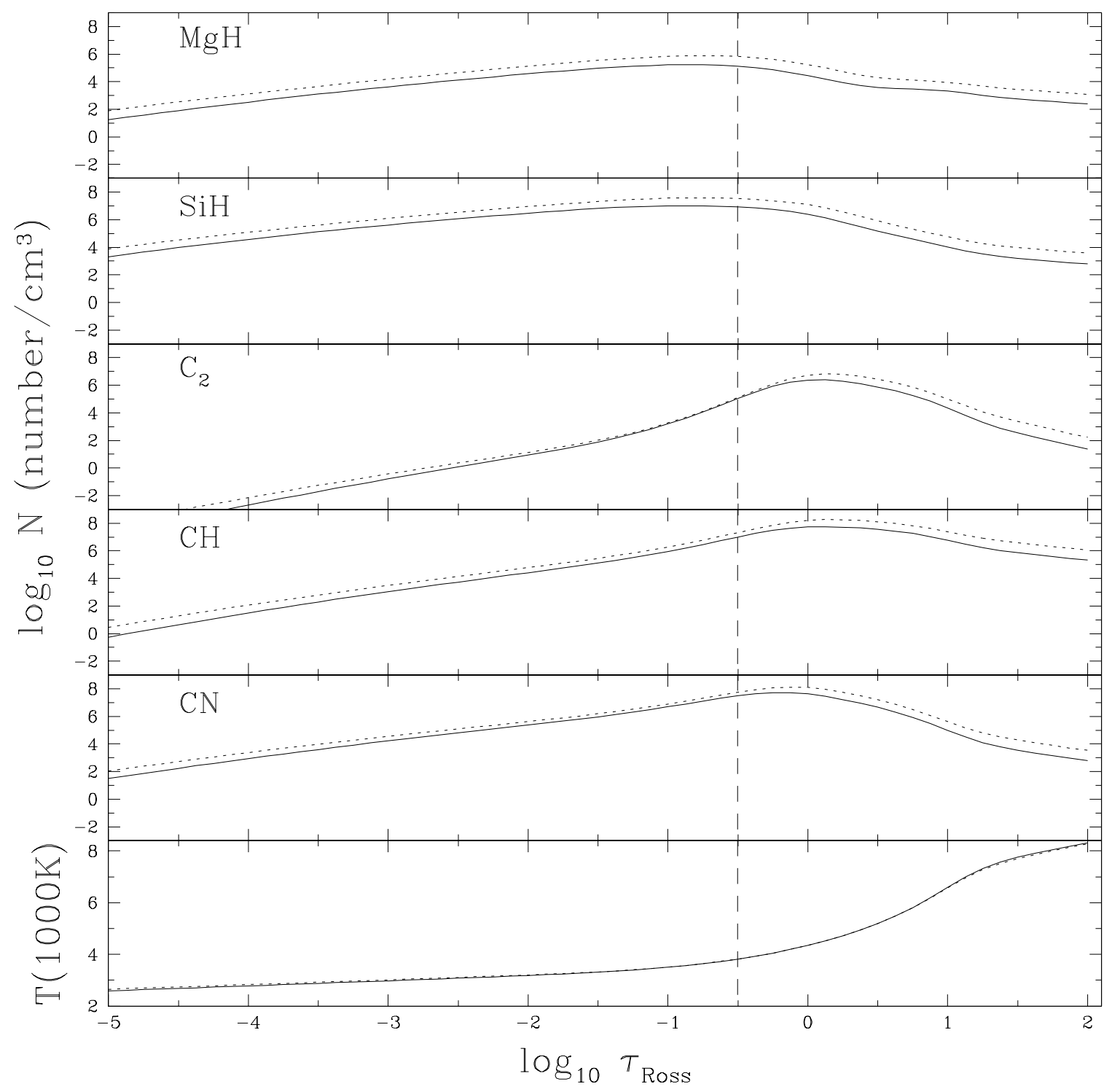

Fig. 7.- Top five panels show the variation of the number density of prominent diatomic molecules with atmospheric depth, represented by $\tau_{\text {Ross }}$, for two red giant spherical atmospheres, both having $L_{\star}=500 L_{\odot}$ and $R_{\star}=50 R_{\odot}$. The solid lines are for $M_{\star}=0.8 M_{\odot}$ and the dotted lines are for $M_{\star}=2.4 M_{\odot}$. The vertical dashed line is at $\log _{10}\left(\tau_{\text {Ross }}\right)=-0.5$, which is the approximate limit of our view into the stellar atmosphere. Bottom panel shows the variation of the temperature with optical depth for the two models, which provides additional context for the depth dependence of the molecular number densities. 


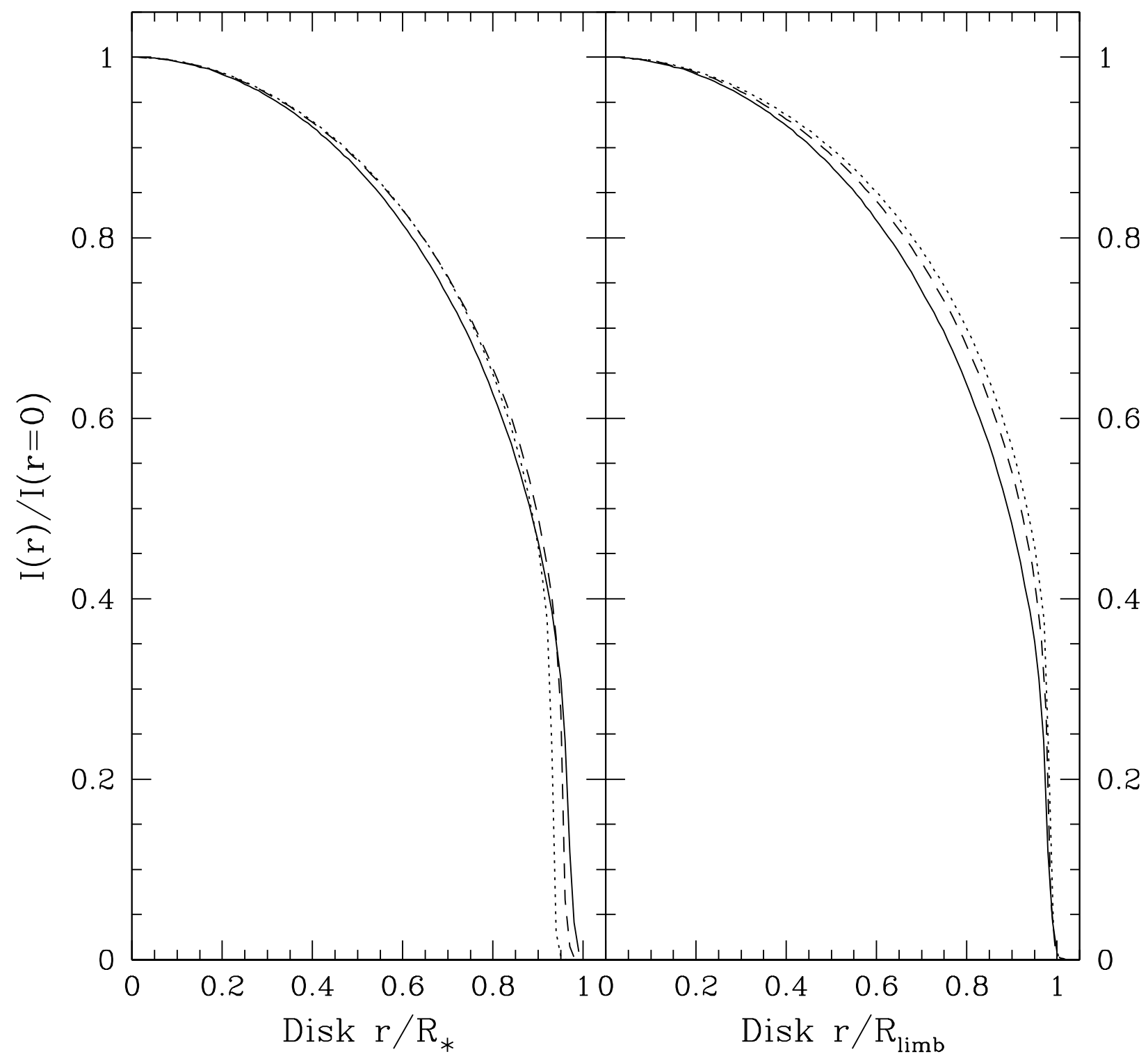

Fig. 8. - The left panel has the surface-brightness distributions plotted as a function of the fractional disk radius, $r / R_{\star}$, for three red giant spherical models, all having the same $L_{\star}, R_{\star}$ and $M_{\star}$. The solid line is the model with solar metallicity, the dashed line is the model with 0.1 solar metallicity, and the dotted line is the model with 0.01 solar metallicity. The right panel plots the same surface-brightness distributions, now as a function of the renormalized fractional radius, $r / R_{\text {limb }}$, defined by equation (44) in $\S 2.1$. The lines have the same meaning as in the left panel. 
with metallicity, with the intensity curve steepening as the composition becomes more metal poor. Therefore, to determine the star's mass from its intensity distribution it is essential to know the star's composition. However, this requirement is much less stringent than what is needed to utilize a particular spectral feature, such as those highlighted in $\S$ 2.2. Instead of requiring a precise value of the composition for the element producing a particular spectral line, only the overall composition of the star is needed, which can be determined by analyzing a selection of spectral features, or perhaps adequately estimated from spectral classification.

\section{Red Supergiants}

To extend our exploration of mass indicators, we next considered red supergiants. Again, we use the SATLAS_ODF program to compute atmospheric models that are spherically symmetric, this time with the representative parameters $L_{\star}=50,000 L_{\odot}, R_{\star}=500 R_{\odot}$ and solar composition. For the masses we chose $M_{\star}=8 M_{\odot}, M_{\star}=16 M_{\odot}$ and $M_{\star}=24 M_{\odot}$. Our final models achieved constancy in the flux and the flux derivative very similar to the red giant models. The fundamental parameters of these models yield $T_{\text {eff }}=3865 \mathrm{~K}$, the same as the red giant models, but $\log _{10}(g)=-0.06$ for the $8 M_{\odot}$ model, $\log _{10}(g)=0.24$ for $16 M_{\odot}$ model and $\log _{10}(g)=0.42$ for the $24 M_{\odot}$ model. Using the relative extension parameter defined in equation (3), the $8 M_{\odot}$ model has $\epsilon(-3) \approx 0.027$ and $\epsilon(-6) \approx 0.065$, the $16 M_{\odot}$ model has $\epsilon(-3) \approx 0.013$ and $\epsilon(-6) \approx 0.031$ and the $24 M_{\odot}$ model has $\epsilon(-3) \approx 0.008$ and $\epsilon(-6) \approx 0.020$, which, again, are all modest extensions.

\subsection{Surface Brightness Distribution}

The surface-brightness distributions of the red supergiants were computed and analyzed in exactly the same way as for the red giants, and the wavelength variation of the half-intensity point determined from the renormalized intensities is plotted in Figure 9, Comparing Figure 9 with Figure 4 it is obvious that the trends and features are very simi-

lar, with just minor difference in detail. As before, the different masses change the surface brightness by a few percent, and for $\lambda \lesssim 900 \mathrm{~nm}$ a spectral resolving power of $R \sim 50-100$ is essential to isolate these regions. For $\lambda>900 \mathrm{~nm}$ the separation between the curves is more constant, and a lower spectral resolving power would be adequate to detect this difference. 


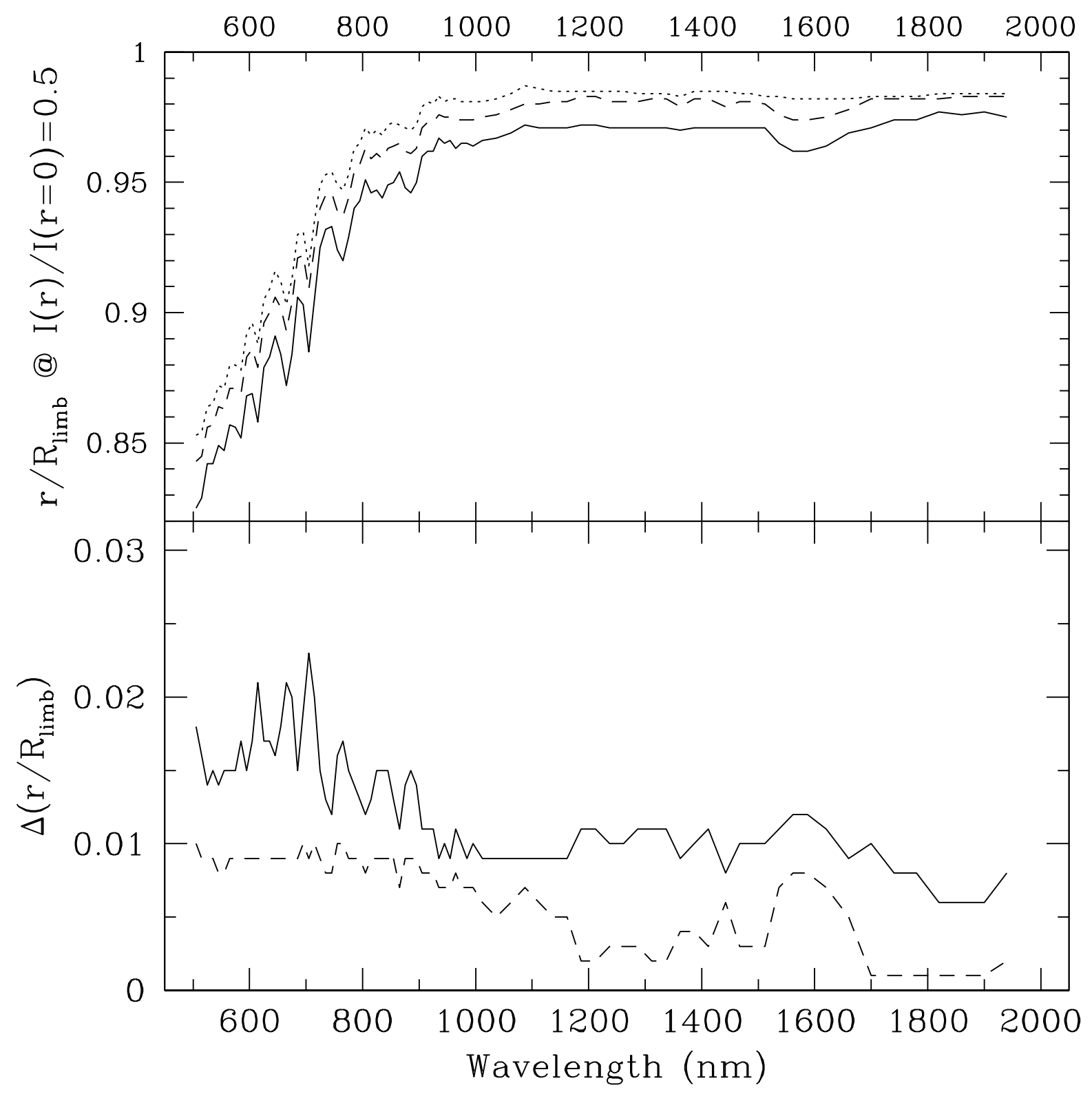

Fig. 9.- Top plot shows for the three red supergiant spherical models the wavelength dependence of the renormalized fractional radius where the surface brightness is half the central brightness. The models all have $L_{\star}=50,000 L_{\odot}, R_{\star}=500 R_{\odot}$ and solar composition. The solid line is the $8 M_{\odot}$ model, the dashed line is the $16 M_{\odot}$ model and the dotted line is the model with $M_{\star}=24 M_{\odot}$. Bottom plot is the difference between the models in the top plot, with the solid line being the difference between the $8 M_{\odot}$ and $16 M_{\odot}$ models and the dashed line is the difference between the $16 M_{\odot}$ and $24 M_{\odot}$ models. 


\subsection{Spectrum}

To search for signatures of mass, we used the spherical red supergiant model atmospheres with $M_{\star}=8 M_{\odot}$ and $M_{\star}=24 M_{\odot}$ to synthesize the flux spectrum from 400 to $1000 \mathrm{~nm}$ at $R=10^{5}$ using the same line lists as for the red giants. Comparing the line depths, we again found that $\approx 95 \%$ of the lines were either unchanged or became weaker. As was found for the red giants, the lines displaying the greatest weakening with increasing mass were located at the shortest wavelengths we computed. The trend found for the red giants, that the amount of weakening decreases with increasing wavelength, also holds true for the red supergiants, but the drop off is somewhat more gradual until the very longest wavelengths. The total variation over the spectral range $400-1000 \mathrm{~nm}$ is the same.

As was found for the red giants, the lines that weaken the most with increasing mass were overwhelmingly ions of heavy elements, with the [O I] lines remaining noteworthy exceptions. The [O I] lines in the supergiants are about twice as strong as for the giants, but their percentage weakening with increasing mass is somewhat less. In addition, the equivalent widths of [O I] $636.3776 \mathrm{~nm}$ and $630.0304 \mathrm{~nm}$ for the red supergiants each weaken by comparable amounts, although the percentage changes are different, but for the red

giants the equivalent width change of the 630.0304 line is almost $50 \%$ greater than for the $636.3776 \mathrm{~nm}$ line.

The $<5 \%$ of the lines that become stronger with increasing mass are all lines of either $\mathrm{MgH}$ or $\mathrm{SiH}$, with the $\mathrm{MgH}$ lines changing by about twice as much as the $\mathrm{SiH}$ lines. Although the $\mathrm{MgH}$ lines of the supergiants are not as deep as they are in the giants, their depths change by about $20 \%$ more than between the most and least massive giant models, and this is also true for the change of their equivalent widths. The variation with depth of the number density of the diatomic molecules for the supergiant atmospheres is very similar to the variation with depth for the red giants shown in Figure 7.

\section{Discussion}

Both the distribution of the surface brightness and the flux spectrum contain indicators of the mass of the red giants and supergiants. Of these, the spectral features of [O I] and $\mathrm{MgH}$ have been recognized and used previously, although they were associated with $\log _{10}(g)$, which depends on the combination of two of the fundamental stellar parameters. With the ability to measure independently both $L_{\star}$ and $R_{\star}$, these lines can now be used to determine the mass of a non-binary star. Current spectroscopic capability is clearly sufficient to measure these lines with the precision required to do the analysis. 
The major complication in using spectral features to determine the stellar mass is the line variability exhibited by some giants and by many supergiants. In an extended observational study of Betelgeuse ( $\alpha$ Ori, HR 2061, HD 39801) Gray (2000, 2001, 2008) recorded variations in the strength of several neutral metal lines in a relatively small spectral window centered on $\lambda 625.0 \mathrm{~nm}$. These variations were attributed to changes in the continuous opacity that alters the contrast between the line core and the adjacent continuum. Three-dimensional radiative hydrodynamic modeling also predicts time variation of the spectrum due to surface inhomogeneities (Chiavassa et al. 2011). The spectral analysis presented here found that most lines, including the neutral lines studied by Gray, have a negligible variation with mass. The specific spectral lines that we identified probably do show the spectral variations caused by surface structure in addition to the dependence on mass. Therefore, to employ the mass-sensitive spectroscopic features found here, it clearly is necessary to normalize the line strengths to adjacent lines insensitive to mass in order to remove the variability caused by processes occurring within the atmosphere. This is in addition to eliminating the effect of the star's composition by normalizing to other lines of the same element, as was done by Bell et al. (1985), Bonnell \& Bell (1993a) and Bonnell \& Bell (1993b).

The signature of mass in the surface-brightness distribution is just a few percent, which is smaller than the variation of the spectroscopic features. However, this small signature may not be as challenging as it appears from our analysis. In our tests the giant and supergiant masses were each varied by a factor of three, resulting in $\Delta \log _{10}(g)=0.48$ in both cases, with the corresponding extension parameter $\epsilon(-3) \lesssim 0.03$ and $\epsilon(-6) \lesssim-0.06$. Even careful, detailed observational analyses of red giants and supergiants frequently have uncertainties $\approx 0.5$ or more in their determinations of $\log _{10}(g)$. Arcturus provides an illustrative example because it has data of the highest quality, which have been analyzed repeatedly. The thorough study by Peterson et al. (1993) concluded that $\log _{10}(g)=1.5 \pm 0.15$, found by minimizing the differences between the observed high resolution flux spectrum and spectra synthesized from a grid of plane-parallel ATLAs stellar atmospheres. The equally thorough examination of Arcturus by Griffin \& Lynas-Gray (1999) determined that $\log _{10}(g)=1.94 \pm 0.05$ by minimizing the differences between observed and computed spectral energy distributions, again using plane-parallel AtLas models. Recently, Ramírez \& Allende Prieto (2011) determined $\log _{10}(g)=1.66 \pm 0.05$ for Arcturus using evolutionary isochrones. The agreement of the $\log _{10}(g)$ values is just marginal considering the error bars. Stars that are less well observed than Arcturus often have significantly larger uncertainty in their $\log _{10}(g)$ determinations. If the surface-brightness distribution could determine $\log _{10}(g)$ with an uncertainty of 0.5 , it would be competitive with the currently employed methods. In addition, the physics responsible for the surface-brightness signature of mass is particularly simple, just hydrostatic and thermal equilibrium. We did find that the intensity distribution depends on the general 
metallicity of the stellar atmosphere, but estimates from spectral classification or a more quantitative determination from a selection of spectral lines would be adequate to determine this parameter. The resulting mass determination will provide a different perspective from the other methods that rely on significantly different and more complex physics.

The measurement of stellar surface brightness distributions is a revolutionary observational achievement, but one that is still developing. For example, the recent study by Lacour et al. (2008) of Arcturus, a star whose disk is well resolved by optical/infrared interferometry, concluded that the disk was not uniformly bright, but was unable to observe exactly how the surface brightness varied with position on the disk. Several studies of Betelgeuse (Buscher et al. 1990; Tuthill et al. 1997; Young et al. 2000; Haubois et al. 2009) have detected large bright features on its surface, while other studies (Ohnaka et al. 2009, 2011) have found only slight deviation from a uniform disk, significantly smoother than the predictions of the three-dimensional radiative hydrodynamic models (Chiavassa et al. 2009). However, when observational agreement is reached about the true appearance of the surfaces of these stars, a key point is that the features will be due to physical processes taking place within the atmosphere. These disk substructures will complicate the observations, but they do not invalidate the conclusion reached here that the basic trend of the stellar surface brightness distribution contains the signature of the fundamental global stellar parameter of mass.

Neilson \& Lester (2011, 2012) have recently explored a different, more statistical way of using the surface-brightness distribution of a cool, luminous star to determine its mass. Using the same SATLAS_ODF code, they computed thousands of spherical model atmospheres with $L_{\star}, M_{\star}$ and $R_{\star}$ thoroughly covering the parameter space of the red giants and supergiants. After computing the surface-brightness distribution for each model, a limb-darkening law with two coefficients was fit to the intensities. An analysis of the ensemble's limb-darkening coefficients showed that they depend on the atmospheric extension, characterized by the parameter $R_{\star} / M_{\star}$. By fitting the limb-darkening law to a star's observed surface-brightness distribution, the coefficients determine the star's $R_{\star} / M_{\star}$. Determining the star's $R_{\star}$ using optical interferometry and parallax measurements gives the star's mass. The approach taken by Neilson \& Lester (2011, 2012) is quite different from the analysis done here, but the conclusion is the same; it is possible to determine the mass of a cool, luminous star by analyzing its surface brightness distribution.

A potential interesting variation of the methods explored here is to apply them to radially pulsating stars, such as Cepheids. Even though Cepheids lose mass over time via a stellar wind (Neilson \& Lester 2008), at a given epoch the Cepheid's mass is constant. By measuring the periodic variation of the star's luminosity and radius, the diagnostics explored 
here would be able to determine the Cepheid's mass at its current evolutionary stage.

\section{Conclusions}

We have used the SATLAS_ODF program (Lester \& Neilson 2008) to compute spherical model stellar atmospheres characterized by the three fundamental parameters $L_{\star}, M_{\star}$ and $R_{\star}$, which were chosen to represent typical red giants and supergiants. Because it is possible to observe $L_{\star}$ and $R_{\star}$, we held the luminosity and radius constant and varied the mass. Searching the radiation of the models for features that varied with $M_{\star}$, we found that there is a systematic change in the surface-brightness distribution, which we characterize by the renormalized fractional radius, $r / R_{\text {limb }}$, at the stellar disk's half-intensity point, $I(r) / I(r=$ $0)=0.5$. For the giant and supergiant models used here, the $r / R_{\text {limb }}$ varies by a few percent for the mass range we explored, which is currently below observational detection. However, with a larger mass variation, and a corresponding larger atmospheric extension, and with improving observational techniques, this signature will become viable.

We also synthesized the flux spectra of the red giant and supergiant spherical models with a spectral resolving power of $10^{5}$, taking into account the spherical radiative transfer. The most interesting changes with mass were found to be the lines of $[\mathrm{O} \mathrm{I}]$, which become weaker with increasing mass, and numerous lines of $\mathrm{MgH}$ in the $X^{2} \Sigma^{+} \rightarrow A^{2} \Pi$ band head around $500 \mathrm{~nm}$, which strengthen with increasing mass. Both of these spectral features have been used previously as indicators of $\log _{10}(g)$, and they can be used now to determine stellar mass with current spectral capabilities.

This work has been supported by a Discovery grant from the Natural Sciences and Engineering Research Council of Canada to JBL, and HN acknowledges funding from the Alexander von Humboldt Foundation. We thank the referee for his/her many detailed com-

ments, questions and suggestions that led us to examine our results more closely and to explain them more clearly.

\section{REFERENCES}

Armstrong, J. T., Mozurkewich, D., Rickard, L. J., et al. 1998, ApJ, 496, 550

Asplund, M., Grevesse, N., Sauval, A. J., \& Scott, P. 2009, ARA\&A, 47, 481

Bell, R. A., Edvardsson, B., \& Gustafsson, B. 1985, MNRAS, 212, 497 
Bessell, M. S. 2005, ARA\&A, 43, 293

Bonnell, J. T., \& Bell, R. A. 1993a, MNRAS, 264, 334

-. 1993b, MNRAS, 264, 319

Buscher, D. F., Baldwin, J. E., Warner, P. J., \& Haniff, C. A. 1990, MNRAS, 245, 7P

Chiavassa, A., Plez, B., Josselin, E., \& Freytag, B. 2009, A\&A, 506, 1351

Chiavassa, A., Pasquato, E., Jorissen, A., et al. 2011, A\&A, 528, A120

Gray, D. F. 2000, ApJ, 532, 487

-. 2001, PASP, 113, 1378

-. 2008, AJ, 135, 1450

Grevesse, N., \& Sauval, A. J. 1998, Space Sci. Rev., 85, 161

Griffin, R. E. M., \& Lynas-Gray, A. E. 1999, AJ, 117, 2998

Gustafsson, B., Edvardsson, B., Eriksson, K., et al. 2008, ArXiv e-prints, 805

Haubois, X., Perrin, G., Lacour, S., et al. 2009, A\&A, 508, 923

Hauschildt, P. H., Allard, F., Ferguson, J., Baron, E., \& Alexander, D. R. 1999, ApJ, 525, 871

Lacour, S., Meimon, S., Thiébaut, E., et al. 2008, A\&A, 485, 561

Lester, J. B., \& Neilson, H. R. 2008, A\&A, 491, 633

Mourard, D., Clausse, J. M., Marcotto, A., et al. 2009, A\&A, 508, 1073

Neilson, H. R., \& Lester, J. B. 2008, ApJ, 684, 569

-. 2011, A\&A, 530, A65

-. 2012, A\&A, 544, A117

Öhman, Y. 1934, ApJ, 80, 171

Ohnaka, K., Hofmann, K.-H., Benisty, M., et al. 2009, A\&A, 503, 183

Ohnaka, K., Weigelt, G., Millour, F., et al. 2011, A\&A, 529, A163 
Partridge, H., \& Schwenke, D. W. 1997, J. Chem. Phys., 106, 4618

Peterson, R. C., Dalle Ore, C. M., \& Kurucz, R. L. 1993, ApJ, 404, 333

Petrov, R. G., \& AMBER Consortium. 2003, Ap\&SS, 286, 57

Ramírez, I., \& Allende Prieto, C. 2011, ApJ, 743, 135

Shayesteh, A., Henderson, R. D. E., Le Roy, R. J., \& Bernath, P. F. 2007, J. Phys. Chem. A, 111, 12495

Tokunaga, A. T. 2000, Infrared Astronomy, ed. A. N. Cox, 143

Tuthill, P. G., Haniff, C. A., \& Baldwin, J. E. 1997, MNRAS, 285, 529

Young, J. S., Baldwin, J. E., Boysen, R. C., et al. 2000, MNRAS, 315, 635 